\title{
Procédé de calcul graphique des cheminées d'équilibre
}

\section{Method of graphic calculation for surge tanks}

\author{
PAR J. CONTE
}

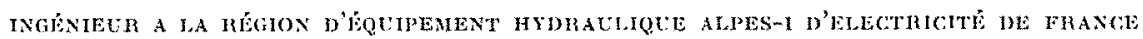

\begin{abstract}
liemplot de la variable qª dans l'étude des cheminces d'étuilibre, eflectué en valeurs relttibes et pur methode graphitue, permet une simplificalion appréciable des épures et en angmente la rapidité d'exécution.

Dans la méthode exposée, la détermination al" la direction moyenne de la nomale ì la conrbe $\mathrm{q}^{2}=\mathrm{f}(\mathrm{x})$ dans un intervalle déferminé permet le trace de ladite courbe en substituant it l'are élémentaire sa corde tracée à l'équerre.
\end{abstract}

L'ingénieur qui étudie le fonctionnement d'une cheminée d'équilibre dispose d'un « outillage » varié et précis. Il peut, en effet, selon ses gonts, user de procédés de calculs numériques, graphiques ou semi-graphiques, lui assurant une précision au moins égale à celle qu'il peut, en toute bonne foi, attribuer aux données du problème.

Notons, en particulier, les surprises qu'apporte la mesure des pertes de charge quand on l'effectue à la mise en service... ou même quand on la vérifie quelques années après.

Le procédé de calcul proposé n’a aucume prétention à l'amélioration d'une précision déjà suffisante, mais son emploi a montré des qualités de rapidité et de commodité non négligeables pour les projeteurs.

Rappelons tout d'abord que MM. Calame et Gaden, introduisant les premiers dans leur "Théorie des chambres d'équilibre » l'emploi des valeurs relatives, montraient que le mouvement oscillatoire créé dans le systìme par une manœuvre pouvait être représenté par l'ensemble des équations :

$$
\begin{aligned}
& \frac{d z}{d q}=-\frac{q}{z+p+r \pm(1 / 2 \pi 0)} \\
& \frac{d z}{d t}=2 \pi q
\end{aligned}
$$

\begin{abstract}
By using the bariable $\mathrm{q}^{2}$ in the graphical methot of surge tank innestigations, based on relative values, an appreciable simplification is mate to the diagrams and the speed at toich they can be produced is increased.

In the mehod described, the metn direction of the nomal to the $\mathrm{q}^{2}=\mathrm{f}(\mathrm{x})$ curve is fired within a given intertal and then the curve itself is built up by ploting the chord of each elemental art perpenticular to the normal.
\end{abstract}

$$
\frac{d q}{d t}=-2 \pi[z+p+r \pm(1 / 2 \pi \theta)]
$$

Nous pensons inutile de rappeler les notations employées qui sont universellement connues.

Les trois équations ne sont pas indépendantes, l'une quelconque pouvant être déduile des deux autres. Seules, les équations (2) et (3) ont une signification physique évidente : l'équation (2) indiquant que la variation de niveau dans la cheminée est due au débil qui y pénètre on qui en sort; l'équation (3) que l'accélération est due à la différence de charge piézométrique entre amont et aval.

L'équation (1) montrant que la sous-normale de la courbe représentant la variation de $z$ en fonction de $q$, a pour valeur l'expression $z+p-+r \pm(1 / 2 \pi 0)$, les auteur's construisaient cette courbe par une série de petits arcs de cercle ayant pour centre théoriquement le point de rencontre de deux normales si elles sont tris voisines, mais en effectuant en réalité une correction estimée, la normale à l'extrémité d'un arc élémentaire ne pouvant être effectivement connue qu'après le tracé de cet arc.

\section{***}

Quelles simplifications peut-on apporter à ce procédé?

En premier lieu, on peut remarquer que les temps sont toujours affectés du coefficiont $2 \pi$ 
et, comme le suggère M. STucky dans son cours sur les cheminées d'équilibre, faire disparaître ce coefficient en changeant l'unité de temps et en adoptant, à cet effet, non pas la période de loscillation, mais cette période divisée par $2 \pi$.

Pour dépouiller plus commodément les études, nous adopterons la notation $n \theta$ pour la durée de la manouvre complète, 0 représentant la duréc effective de la manœuvre réalisée, $n$ un coefficient de majoration si la manouvre n'est que partielle.

Si, d'autre part, nous divisons les deux membres de l'équation (1) par $2 q$, le numćrateur du deuxième membre de l'équation devient la constante $1 / 2$, le dénominateur du premier membre devient $2 q d q$, ce qui est la difórentielle de $q \stackrel{ }{*}$.

Dans ces conditions, les équations deviennent :

$$
\begin{aligned}
& \frac{d z}{d \ell^{2}}=-\frac{1 / 2}{z+p+r+(1 / n \theta)} \\
& \frac{d z}{d t}=q \\
& \frac{d q}{d t}=-[z+p+r \pm(1 / n \theta)]
\end{aligned}
$$

L'équation (4) montre que la normale à la courbe $z=\varphi\left(q^{2}\right)$ a pour pente :

$$
-\cdots \frac{z+p+r \pm(1 / n 0)}{1 / 2}
$$

La tangente à la courbe $q=\psi(t)$ a une pente moitié. Mais si nous prenons pour les temps une échelle moitié et un sens convenable de l'axe, nous pouvons rendre ces deux pentes égales.

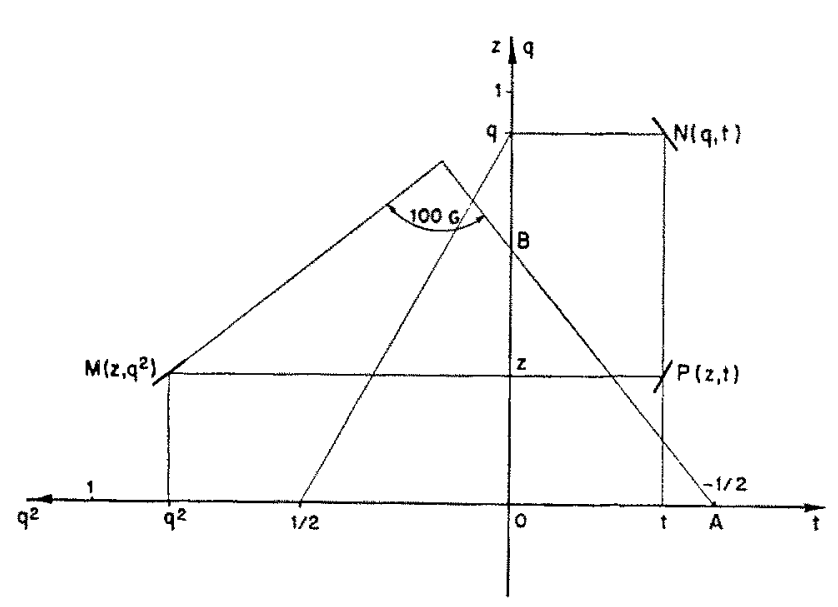

Fis, 1.

Pour nous résumer, traçons un graphique comportant deux axes rectangulaires (fig. 1). Utilisons la moitié gauche de la figure pour représenter la variation de $z$ en fonction de $q^{2}$ en portant sur l'axe vertical les valeurs de $z$ et sur l'axe hori- zonlal les valeurs de $q^{2}$. Ulilisons la moitic droile de la figure pour représenter les variations de $z$ et $q$ en fonction du temps en portanl sur l'axe horizontal les temps à l'échelle $1 / 2$ el sur l'axe vertical à la fois $z$ et $q$.

Soit un point $M$ de coordonnćes $\left(z, q^{\prime}\right)$ de la moitic gauche de la figure el ses homologues $\mathrm{N}$ de coordonnèes $(q, t)$ et $P$ de coordonnées $(z, t)$ de la moitié droite.

Traçons une droite joignant le point $A$ de l'axe horizontal d'abscisse - 1/2 sur l'échelle $q^{\prime \prime}$ (c'esta-dire 1 sur l'ichelle des temps) au point $B$ de l'axe vertical et d'ordonnée $z+p+r \pm\left(1 / n^{0}\right)$.

La tangente en $M$ a la courbe lieu du point $M$ lui est perpendiculaire.

La tangente en $\mathrm{M}$ à la courbe lieu du point $\mathrm{M}$ lui est parallèle.

La langente en $P$ à la courbe lieu du point $P$ est parallèle à la droite joignant le point $(z==0, q=1 / 2)$ au point $\left(q^{2}=0, z=q\right)$.

L'avantage oblenu semble se réduire à une relation de perpendicularité entre les tangentes en $\mathrm{M}$ et en $\mathrm{N}$.

Mais supposons que nous ayons déterminé sur le graphique que nous venons de décrire un point $M$ et que la direction de la normale en $M$ soit AB.

Supposons (fig. 2) que nous puissions tracer une courbe $C$ pour tous les points de laqualle la valeur de l'expression $z+p+r \pm(1 / n 0)$ soit constante, soit $\alpha$.

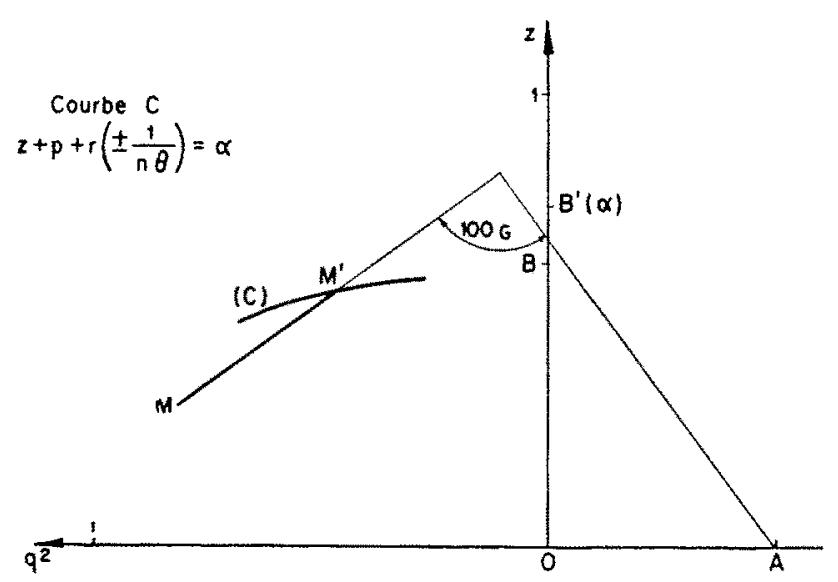

Fic. 2.

La courbe lien des points tels que $M$ coupera celte courbe en un point $\mathrm{M}^{\prime}$, la tangente en $\mathrm{M}^{\prime}$ étant perpendiculaire à la droite de référence joignant $A$ an point $B^{\prime}$ d'abscisse $O$ et d'ordonnée $\alpha$.

La langente en $M$ est perpendiculaire à $A B$.

La tangente en $\mathrm{M}^{\prime}$ est perpendiculaire à $\mathrm{AB}^{\prime}$. Ia pente moyenne de Ia langente entre $\mathrm{M}$ el $\mathrm{M}^{\prime}$, 


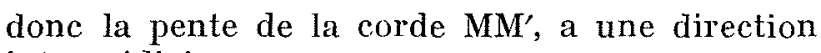
intermédiaire.

Nous admettrons que cette corde est perpendiculaire à la droite de référence joignant $\mathrm{A}$ au milieu de $\mathrm{BB}^{\prime}$. Autrement dit nous prenons comme droite de référence pour l'intervalle MM' la médiane du triangle $B A B^{\prime}$. Ceci est d'autant plus vai que l'intervalle BB' est réduil, ce dont l'opérateur reste maitre. L'allure régulière et à faible courbure des courbes rend d'ailleurs superflue une précaution exagérée en cette matière.

Nous pouvons faire deux remarques :

- En premier lieu, la pente de la droite de référence est proportionnelle à l'expression $z+p+r \pm(1 / n 0)$ qui comporte deux termes : $z$ et $r=\mathrm{Rr}_{\mathrm{q}} q^{2}$ qui sont forcément déterminćs en tout point du sraphique, un terme $\left.1 / n^{0}\right)$, qui est une constante de l'općration, et un terme $p$, fonction du débit en galerie, et qui n'est pas forcément déterminé par la simple position du point $M$ sur le graphique.

- En second lieu, nous obtiendrons une droite de référence de même pente en portant la somme d'une partie seulement des termes $z$, $p, r, \pm(1 / n 0)$ sur l'axe vertical et en joignanl le point obtenu au point sur l'axe d'abseisse -.- 1/2 el ayanl pour ordonnće la somme des autres termes changée de signe.

$$
*
$$

Le principe de notre calcul graphique étant posi, comment se traduit sa mise en cuvre?

Considérons en premier lieu le cas d'une termeture instantanée et soit un aménagement comportant une chambre d'écuilibre avec un étranglement créant pour le débit de plein régime une perte de charge $r_{0}$, placée à l'extrémité d'une galerie où la perle de charge pour ledit débil de plein régime est $p_{0}$.

Dìs la fermeture, le débit entrant dans la chambre est égal au débit passant dans la galerie el nous, avons donc pendant loul le mouvement oscillatoire :

$$
\begin{aligned}
& p=p_{0} q^{2} \quad \text { et } \quad r=r_{0} q^{2} \\
& \text { done } p+r=\left(p_{0}+r_{0}\right) q^{2}
\end{aligned}
$$

La droite joignant le point $q^{2}=1, z=-\left(p_{0}+r_{0}\right)$ à l'origine a en chaque point pour ordonnée $z=-\cdots-(p+r)$, donc pour équation $z+p+r=0$.

Les droites $z+p+r=\mathrm{C}^{\text {te }}$ lui sont paralleles. D'où la construction (fig. 3).

Mener depuis le point correspondant à l'origine du mouvement (soit $z=-p_{0}, q^{2}=1$ ) une première parallèle à la droite $z+p+r=0$, puis une série d'autres parallèles à intervalles quelconques.

Joindre le point $A$ aux milieux des intervalles découpés par ces parallèles sur l'axe des $z$ et constituer un faisceau de droites de référence. Puis, en partant du point origine, tracer bout à bout une suile de segments de droite délimités par les diverses parallèles tracées et chacun perpendiculaire à la droite de référence correspondante.

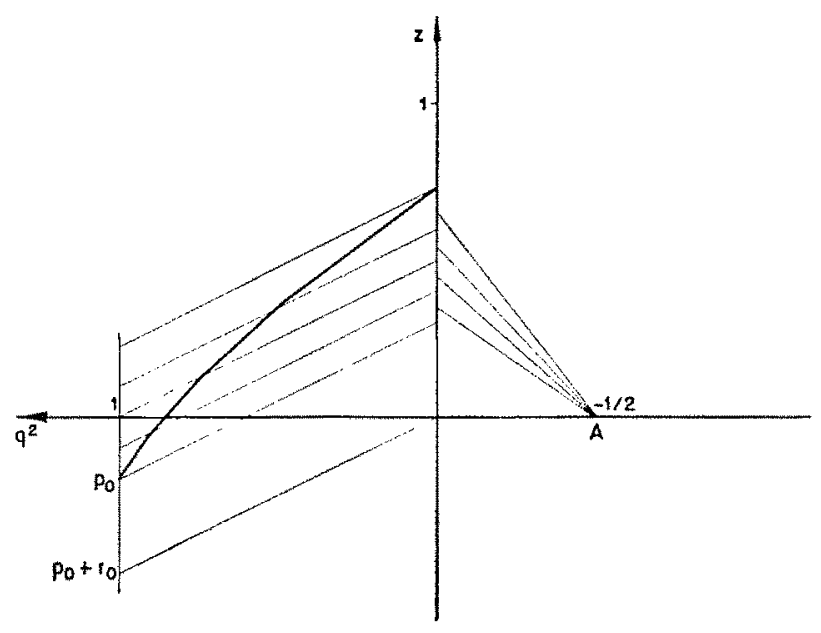

Frc. 3.

L'étude de l'oscillation dans le temps n'a pas beaucoup d'intérèt en cas de mancuvre instantance, elle n'a pas été construite sur la figure 3 pour ne pas surcharger la figure, mais elle ne complique pas beaucoup l'épure, comme le montre la figure 4 tracée avec les mêmes donnés.

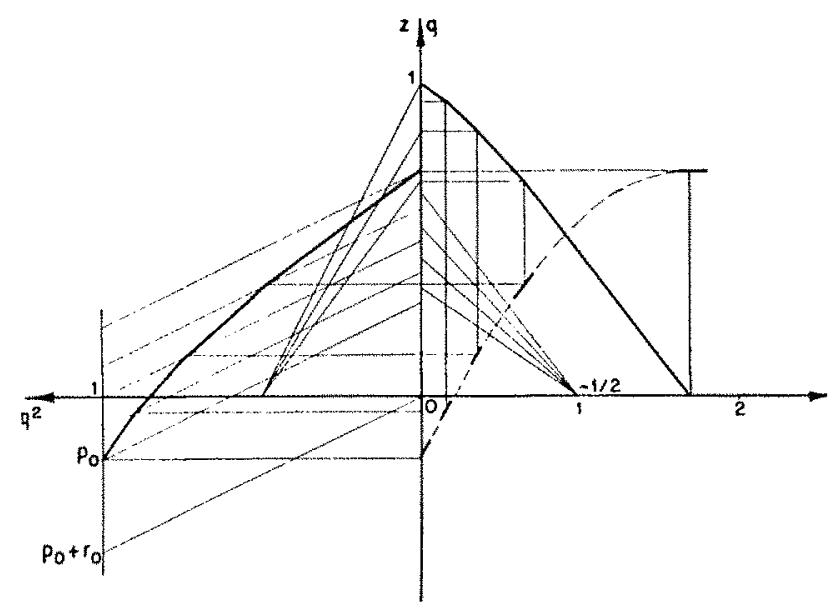

Fili, 4.

Si la chambre ne comporte pas d'etranglement, Ja courbure à l'origine est plus prononcée. Le rayon de courbure, toujours égal $-1 / 2$ en ce point, permet de sortir de la zone oì le tracé risquerait d'être plus délical (fig. 5).

Si la cheminée est déversante, on peut utiliser la methode usuelle, c'est-a-dire chercher 
l'intersection de la courbe $z=\left(q^{2}\right)$ avec la courbe de déversement $\gamma^{2}=\left(z-z_{0}\right)^{3}$, mais ce procédé, négligeant le début du déversement, donne une cote et un débit maximum exagérés. On peut

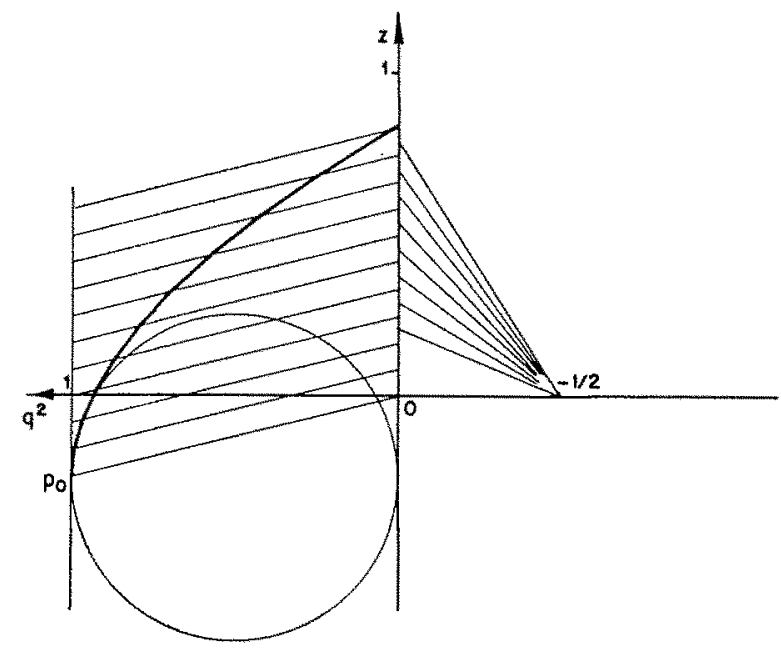

Fia. 5.

également procéder à des corrections. Le calcul montre en effet que l'origine de la droite de référence pour un point de la zone de déversement n'est plus A mais un point plus rapproché de l'origine et d'abscisse $-1 / 2(1-\omega)$, $\omega$ étant le rapport du débit déversé au débit entrant dans la chambre.

On obtient un résultat très acceptable en prenant la valeur moyenne entre $\omega=0$, origine du déversement, et $\omega=1$, fin du déversement, c'est-

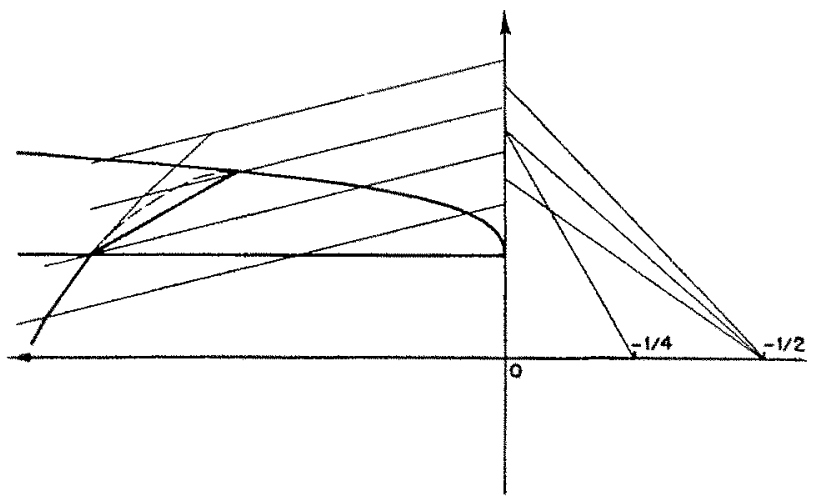

Fia, 6 .

à-dire en prenant une droile de référence passant par le point d'abscisse - 1/4 (fig. 6).

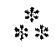

Celle simplicité de construction disparait évidemment quelque peu dès que l'on quitle le cas d'une fermeture instantanée.

Dans les manœuvres brutales d'ouverture, $r$ est toujours fonction linéaire de $q^{2}$, mais $p$ est égal à $p_{0}(1-q)^{2}$ et la courbe représentant $p$ est une parabole. La courbe $\left(p+r=\psi\left(q^{2}\right)\right.$ est également une parabole.

Traçons une série de paraboles parallèles (fig. 7).

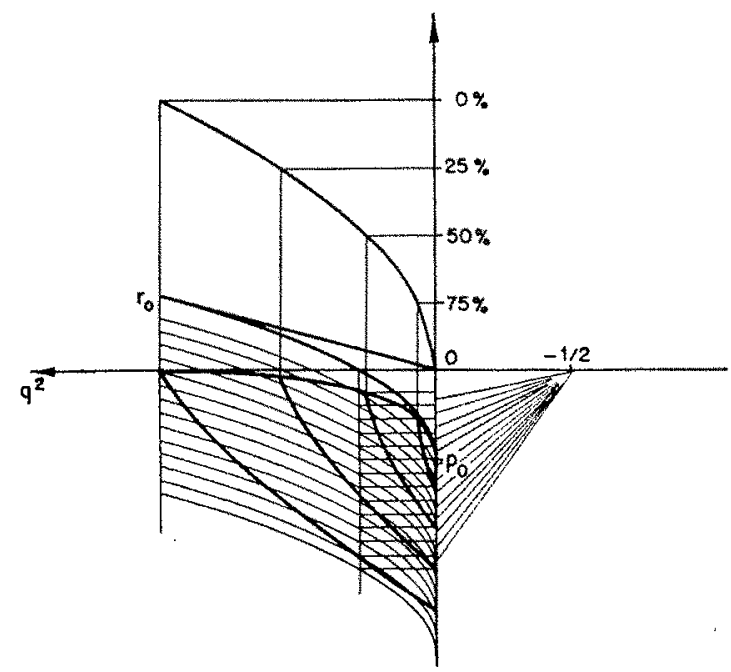

FIG. 7 .

L'opération est évidemment plus compliquée que le tracé de droites parallèles. Elle est toutefois moins difficile qu'il ne parait et quand le dessinateur a trouvé la partie de son pistolet qui convient, il la repère et trace sans difficulté une série de courbes parallèles.

Mais une fois cette mise en place réalisée, il peut tracer par le mème processus que dans l'épure de fermeture simultanément autant de courbes qu'il le désire, partant chacune d'une ouverture initiale différente.

Remarquer que la valeur de $(z+p+r)$ est à prendre par l'écart de chaque parabole avec la parabole $z+p+r=0$, c'est-à-dire sur leur intersection avec la verticale passant par le point de rencontre de la parabole origine avec l'axe des $q^{2}$.

\section{*}

Attaquons-nous enfin au cas le plus complexe: une ouverture partielle progressive.

Construisons une épure avec les deux axes de coordonnces déjà définis (fig. 8) el traçons sur celte épure la loi de manœuvre supposée linéaire cn ulilisant l'axe des $q$ du côté négatif.

Menons par l'origine une parallèle à la droite représentative de la Loi de Manœuvre. Elle coupe l'axe d'abscisse $t=1$ en un point $C$ d'ordonnée $-(1 / n \theta)$.

Soit $\mathrm{N}$ un point de coordonnées $(l, q)$, NG sa distance à la droite que nous venons de tracer. L'ordonnée de G représente l'augmentation depuis le début de la manœuvre du débit absorbé 
se décomposant en une partie NG fournie par la galerie, le solde représenté par l'ordonnée de $\mathrm{N}$ étant fourni par la cheminée d'équilibre.

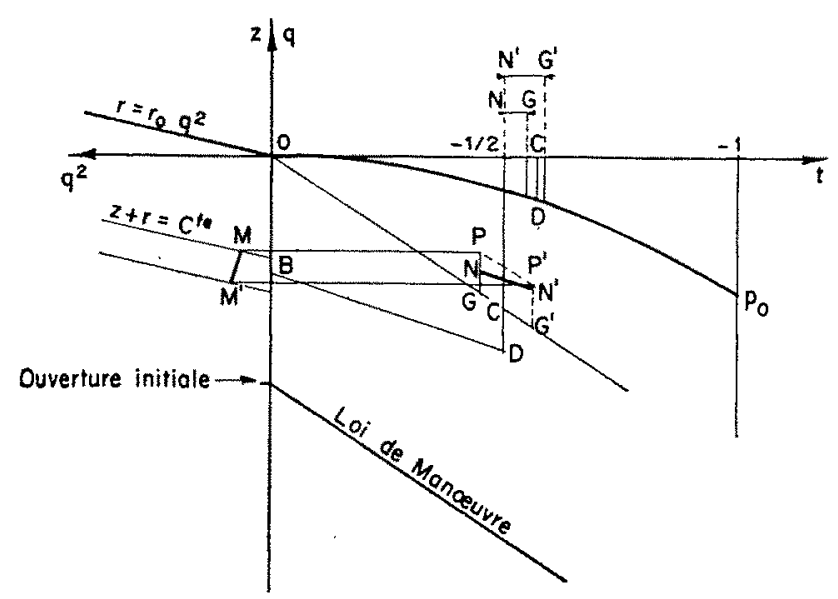

FIG. 8.

Nous pouvons tracer sur notre épure un graphique donnant les pertes de charge en galerie en fonction du débit qui y circule, ce qui nous permet de suivre la variation de $p$ en fonction de la variation de la distance $N G$.

Pour construire notre épure, nous utiliserons une remarque déjà faite et porterons, grâce aux. droites $z+r=C^{\text {te }}$, les valeurs de $z+r$ sur l'axe vertical, landis que nous porterons la valeur de $-p+(1 / n 0)$ sur l'axe $q^{2}=-1 / 2$ (ou $\left.t=1\right)$, ce qui nous donnera un point $D$ situé à la distance $p$ audessous de C déjà tracé et d'ordonnée - $(1 / n \theta)$.

La diffeullé vient de lignorance dans laquelle uous sommes de la valeur moyenne de $p$ dans l'intervalle qui nous conduira du point $\mathrm{M}\left(z, q^{2}\right)$ au point suivant $M^{\prime}$. Nous ne pouvons en connaitre que sa valeur au point $M$. Si les intervalles sont lrès serrés, nous pouvons adopter celte valeur, mais il vaut mieux operer de la facon suivante.

Nons ne construisons pas un point isolé, mais nous suivons la variation d'un point répondant à certaines conditions. Pour obtenir le point $\mathrm{M}$, nous avions utilisé un point tel que $\mathrm{D}$ soit $\mathrm{D}^{\prime}$ succédant lui-même à un point antérieur $\mathrm{D}^{\prime \prime}$. Nous adopterons comme valeur approchée de la correction nous menant de $D^{\prime}$ à $\mathrm{D}$ la correction corrigée qui nous avait menés de $D^{\prime \prime}$ à $D^{\prime}$.

Avec celte valeur approchée nous faisons une opération « à blanc $»$. Comme ces épures se tracent sur papier quadrillé ou mieux millimétré, léquerre mise en place sur la position approchée qui vient d'être définie nous permet de lire la position approximative du point $\mathrm{M}^{\prime} \mathrm{el}$, avec un rappel sur le prolongement Jui-même approximatif du lieu de $N$, d'avoir une valeur approchée de $p$ en fin d'intervalle et par suite une valeur moyenne améliorée d'après laquelle nous faisons le lracé.

En résumé, la mise en place approchée de notre équerre nous a permis d'en corriger la position.

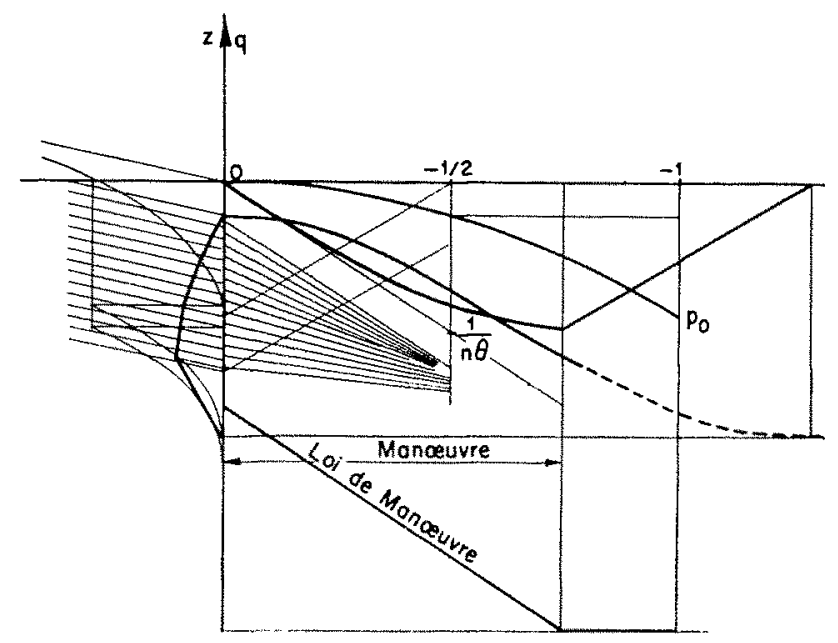

FI(x. 9,

La figure 9 montre l'épure complète ainsi réalisée. Bien entendu, dès que l'on atteint la fin de manouvre, on reprend la construction exposéc pour la manouvre instantanée.

\section{I S C S S I O N}

Présidenl: M. Maurice Galmer.

M. le Président remercie vivement M. Conte de son très intéressant exposé. Il se demande toutefois si, au cours de ce calcul si intéressant par sa rapidite, on ne risque pas de perdre de vue le déroulement du phénomène physique, ce qui est toujours fort ulile pour se proteger contre les erreurs possibles.

M. Conte reconmaît que si ce procédé de calcul est beauenup plus rapide que les épures usuelles tant en valeurs relatives qu'en raleurs réelles, il est cortainement moins parlant, car d'une parl, les courbes en $q^{3}$ sont plus aplaties que les courbes en $q$ trés arondies; d'autre part les troncons de courbe ascendants el descondants restent d'un même coté de l'axe des $Z$.

On pent supprimer le dennier de ces inconvénients en adoptant la fiction d'un $q^{2}$ ayant le signe de $q$ c'est-adire le remplacer par $q \times|q|$

Quand loscillation s'amortit, pour los lres petites valeurs de $q$, la méthode est assez imprecisc, comme les autres d'aillours.

\section{喿}

M. Conte donne lecture de la note ci-apres, remise pay M. Bovvaro qui n'a pu assister a la sćance : 
* Comme le dit son auteur lümmene, la methode exposée se déduit de la méthode Calame el Gaden, en atilisant comme variable $q^{*}$ au lieu de $q$, pour le trace.

«On peut considerer que les résultats de la méthode graphique de Calame et Gaden sont de deux ordres :

« $1^{\circ}$ Is ont permis notamment de tracer des abaques dont l'mportanee est très grande, et qui sont conramment utilisés par les projeteurs de cheminéc.

« 2" Pour les études de cas qui n'entrent pas daus ces abaques, et qui sont certainement un peu moins simples, on peut se deniander si la méthode n'esl pas un peu compliquee, ou au moins an peu artificielle.

«Le fonctionmement des cheminees esl en effet io suivant :

« a) Elles assurent, par la suppression (ou la dépression) qu'elles créent, une accélération de l'eau dans la galerie. C'est, en particulier, le rôle exclusif de l'étranglement.

«b) lan attendant que le débit de la gaierie égale celui qui est demandé, elles assurent le stockage et lo déstockage des caux correspondant à cette diffe. rence.

«Les équations qui régissent ces deux phénomènes, séparés, sont extrềmement simples : c'est $H^{*}=m \gamma$ pout la première, et l'équation de continuité pour la seconde.

«L'inconvénient de toute méthode analytique est de les synthétiser par une seule opération, beaucoup plus compliquée que les opérations individuelles indiquées précedemment. Il est alors beaucoup plus fitcile de se tromper.

« D'autre part, les mancurres non instantanées, arec des changements de section, sont beacoup plus difficiles à étudier.»

M. Conte répond que le procédé qu'il a décrit se déduil cn effet de la méthode Calime et Gaden, que ces auteur ont effectivement tracé des abaques, malheureusement à échelle assez réduite, et dont les projeteurs aiment bien contrôler les indications. Cette vérification ne demande, avec la méthode proposéc, guère plus de 10 minutes pour une termeture instantanée.

Le fonctionnement des cheminés d'équilibre est bien regi par deax lois : une equation de continuite, uno equation exprimant que l'accélération du mourement est due a la différence des charges piézométriques aux deux extrémités du système.

Les différents procédés de calcul utilisent ces equations, soit simultanément (différences finies, Calame of Gaden), soit séparćment (différences finies simplifiées, Schoklitsch).

II. Conte pense préférable l'utilisation simultanée, ec que la méthode qu'il propose réalise en employant directement dans chaque intervalle les valeurs moyennes des diverses grandeurs, exception faite toutefois, dans le cas des manouves temporisées, de la perie de charge en galerie qu'il faut estimer, puis corriger.
Dans les cas très compliqués, il faut recourir à la méthode par differences finies on reeherehant les valeurs moyennes par lâtonnement et en excluant les méthodes simplifies, qui recherehent une compensation en prenant pour certaines grandeurs les valeurs de debut, pour les autres les valeurs do fin d'interralle.

\section{*}

M. Ransfons indique la façon dont il a calcule une cheminée d’équilibre suivant une méthode numérique el au moyen dine machine a cartes perforés 1.B.M.:

Il s'est agi d'une cheminéc d'équilibre implantée sur un canal de fuite mesurant quelque 300 metres de long. Dans ce cas, l'influence de l'énergie cinétique à la base de l'ouvrage jouait bien entendu un role préponderant Nous avons été amenés à introduire dans nos calculs des formules inspirées alors de la récente thèse de M. Gardel formules qui permettaient de tenir compte des différences de charge existant entre la base de la cheminee el la conduite. D'autre part, la forte pente que la courbe de rendement accusait pour le régime considére rendait absolument nécessaire la mise en écuation de cette courbe. Enfin, il y avait lieu de tenix compte de la perte de charge dans la conduite forcée.

En tout, il $y$ a à résoudre une huitaine d'equations nol linéaires à coefficients variables suivant le sens de l'écoulement. Sur la machine, la solution complète a été obtenue pour quatre projets differents, chaque solution comportant en partiealier la détermination de l'amplitade limite des oscillations entretenues (car la cheminéc était instable), ceci au cours d'un intervalle de temps de trois heures.

M. le Président souligne la triple concurrence actuelle - et très profitable à son avis - entre les méthodes griphiques, les machines à calculer et, dans bien des cas, les modèles réduits eux-mémes; c'est ainsi que, pour le canal de la Durance, où se trouve l'usine de Jonques, on peut hesiter entre le modele réduit et la machine à caleuler.

M. Reuenienas demande à M. Conte si son ejpure se prête bien à des calculs de manouvres combinées : par exemple, une fermeture, provoquant des oscillations di plan d'eau dans la cheminée et suivie d'une ouverture a un moment choisi daus les conditions les plus defayorables; c'est un des cas où les abaques de Calame ef Gaden ne sont pas direetement applicables.

M. Conte répond qu'il ne peut répondre a priori paree qu'il n'a pas appliqué la methode à ce cas; cependant son extrême rapidité - l'épure d'ouverture temporiséo demande quarante minutes - semble placer la méthoric dans de bonnes conditions poux cette application. II réalisera l'épure correspondante et la communiquera í M. REMENIERAS.

N. Remenieras estime que la méthode de $M$. Con'r permetra, grâce à sa rapidite, de calculer le fonctionnement de la cheminée dans des cas plus nombreux et plus Faries qu'on n'a coutume de le faire, du fait de la longucur des calculs nécessités par les méthodes usuelles. 